\title{
Novel model for the in vivo study of central nervous system infection due to Acanthamoeba spp. (T4 genotype)
}

\begin{abstract}
Correspondence
Naveed Ahmed Khan

naveed.khan@nottingham.ac.uk
\end{abstract}

Received 28 July 2008

Accepted 22 December 2008

\author{
Parisa Nakhostin Mortazavi, ${ }_{1}^{1}$ Graham Goldsworthy, ${ }^{1}$ Ruth Kirk ${ }^{2}$ \\ and Naveed Ahmed Khan ${ }^{1,3}$
${ }^{1}$ School of Biological and Chemical Sciences, Birkbeck, University of London, London, UK
${ }^{2}$ School of Life Sciences, Kingston University, Kingston upon Thames, Surrey, UK
${ }^{3}$ School of Veterinary Medicine and Science, University of Nottingham, Sutton Bonington, England, UK

\begin{abstract}
In this study it was shown for what is believed to be the first time that the African migratory locust can be used as a model for the study of Acanthamoeba pathogenesis. Mature adult locusts were injected intra-abdominally with $10 \mu$ suspension of $10^{6}$ Acanthamoeba (a clinical isolate of the T4 genotype) in culture medium, or with the same volume of sterile culture medium. Locusts injected with Acanthamoeba showed significant weight loss and reduced production of faeces compared with control locusts. Furthermore, injection of amoebae killed all of the locusts within 17 days at room temperature, although the speed of kill was temperature and dose dependent. When samples of faecal pellets and various tissues of infected locusts were cultured on non-nutrient agar plates containing bacterial lawns, live amoebae were recovered from haemolymph, flight muscle and fat body samples, but not from faeces. When brains dissected from locusts were incubated with an anti-amoebic drug (100 $\mu \mathrm{M}$ chlorhexidine) to kill extracellular amoebae, and then washed, homogenized and cultured on bacteria-seeded non-nutrient agar plates, only lysates from amoebae-infected locusts were positive for Acanthamoeba. This strongly suggests that amoebae invade the locust brain and, indeed, trophozoites of Acanthamoeba could be identified within the brain in histological sections of brains from infected locusts, but not from uninfected locusts. These findings support the view that locusts can be used as a model for the study of Acanthamoeba pathogenesis in vivo.
\end{abstract}

\section{INTRODUCTION}

Members of the genus Acanthamoeba are the causative agents of granulomatous encephalitis, a fatal subacute to chronic disease, and amoebic keratitis, a painful sightthreatening infection. Acanthamoeba spp. are also responsible for cutaneous lesions and sinusitis in AIDS patients (Schuster, 2002; Marciano-Cabral \& Cabral, 2003; Schuster \& Visvesvara, 2004; Khan, 2007).

Although generally considered a rare infection, Acanthamoeba encephalitis is a major concern in human health, in particular with the increasing populations of AIDS patients as a result of human immunodeficiency virus (HIV) type 1 infection worldwide (estimated by the World Health Organization to be 33 million, as of 2007). The approximate rate of death due to Acanthamoeba encephalitis is estimated to be at least 1.57 per 10000 HIV/AIDS deaths (Khan, 2006). A distressing aspect is that Acanthamoeba

Abbreviation: CNS, central nervous system. encephalitis is almost always fatal, despite advances in antimicrobial chemotherapy (Khan, 2007). This is due to an incomplete understanding of the pathogenesis and pathophysiology of the disease. Haematogenous spread followed by blood-brain barrier penetration is thought to be the likely route of invasion of the central nervous system (CNS) by amoebae. However, the precise mechanisms associated with Acanthamoeba invasion of the intravascular space, evasion of the host immune defences and traversal of the blood-brain barrier in vivo require investigation. Current studies in vivo have relied on vertebrate models to study Acanthamoeba encephalitis. Although physiologically relevant, mammalian models are expensive, not routinely available in many laboratories, require labourintensive management and have ethical implications. For these reasons, the use of Locusta migratoria, the African migratory locust, as a model for the study of Acanthamoeba pathogenesis was tested in this study. Locusts are routinely reared in our laboratories and can be handled with ease, as well as injected with large volumes, and a significant 
amount of haemolymph can be collected for assays. Insects rely for their protection against infection on an entirely innate immune system, so the use of an insect model is particularly relevant in the study of Acanthamoeba encephalitis, the control of which has significant dependency on the innate immune system. The overall aim of this study was to determine the feasibility of locusts as a model for the study of Acanthamoeba infection in vivo.

\section{METHODS}

Locusts. African migratory locusts were fed on bran, wheat seedlings and fresh grass. Adult locusts live an average of 80-100 days, and the adult males used in the present study were between 15 and 30 days old with a fully developed innate immune system (Goldsworthy et al., 2003a, b; Mullen \& Goldsworthy, 2003). In initial mortality studies, infected locusts were maintained in the laboratory at room temperature $\left(\sim 20{ }^{\circ} \mathrm{C}\right)$, but in subsequent experiments infected and control locusts were housed in a separate holding room in which the temperature was maintained at $\sim 30{ }^{\circ} \mathrm{C}$.

Culture of Acanthamoeba. A clinical isolate of Acanthamoeba castellanii of the T4 genotype was used (ATCC 50492). Acanthamoeba was routinely grown without shaking in $10 \mathrm{ml}$ culture medium $[0.75 \%$ $(\mathrm{w} / \mathrm{v})$ proteose peptone, $0.75 \%(\mathrm{w} / \mathrm{v})$ yeast extract and $1.5 \%(\mathrm{w} / \mathrm{v})$ glucose] in T-75 tissue culture flasks at $30{ }^{\circ} \mathrm{C}$ as described previously (Khan et al., 2000), with medium refreshed $24 \mathrm{~h}$ prior to experiments. This resulted in more than $95 \%$ amoebae in the trophozoite stage.

Assays to determine parasitaemia and mortality. Locusts were allocated randomly into groups of ten, except where indicated otherwise. Each experimental locust was injected with $10 \mu \mathrm{l}$ culture medium containing $10^{3}-10^{6}$ amoebae into the haemocoel of the locust abdomen by inserting the needle into the intersegmental membrane between two abdominal terga as described previously (Khan \& Goldsworthy, 2007). Mortality was recorded every $24 \mathrm{~h}$, when any dead locusts were removed to prevent cannibalism. Control locusts were injected with $10 \mu \mathrm{l}$ culture medium alone.

To determine whether locusts developed parasitaemia, haemolymph was collected at various intervals after infection. Briefly, the cuticle and arthrodial membrane at the base of one hind leg was swabbed with cotton wool moistened in $70 \%$ ethanol. After allowing the cuticle surface to air dry, a small puncture was made in the membrane using a sterile needle and $5 \mu \mathrm{l}$ haemolymph was collected into calibrated glass capillaries. The haemolymph was inoculated onto non-nutrient agar plates seeded with Escherichia coli K-12. Plates were incubated at $30{ }^{\circ} \mathrm{C}$ and observed daily for the presence of Acanthamoeba.

CNS invasion assays in vivo. To determine Acanthamoeba association with the CNS, locust brains were isolated as described previously (Khan \& Goldsworthy, 2007). Briefly, at various intervals, a number of infected locusts were killed, the left side of the head was removed by a sagittal cut and the brain was dissected out using fine forceps. Care was taken to ensure that both cerebral ganglia were present, and that they were free of fat body tissue and air sacs, and surgical instruments were surface sterilized with methylated spirit between dissections. Each brain was washed by placing it in a microcentrifuge tube containing $1 \mathrm{ml}$ PBS. Tubes were centrifuged by a brief pulse at $750 \mathrm{~g}$ to sediment each brain and the supernatants were discarded. This washing process was repeated three times. Finally, brains were incubated with chlorhexidine (final concentration $100 \mu \mathrm{M}$; Sigma) at $37{ }^{\circ} \mathrm{C}$ for $2 \mathrm{~h}$ to kill extracellular amoebae. The brains were then washed a further three times to remove the chlorhexidine, physically disrupted with sterile tips and inoculated on bacteria-seeded non-nutrient agar plates.

Histological studies. Locusts were injected with $10^{6}$ amoebae and maintained at $\sim 30{ }^{\circ} \mathrm{C}$ for 7 days, after which they were decapitated and the whole heads were fixed under vacuum in $10 \%$ neutral buffered formalin for $72 \mathrm{~h}$. The brains were then dissected as described above and placed in fresh neutral buffered formalin for $48 \mathrm{~h}$ at room temperature. Fixed brains were dehydrated, cleared in Histo-Clear (National Diagnostics), and infiltrated and embedded in Paramat (Gurr). The brains were sectioned serially ( $5 \mu \mathrm{m}$ thickness) and the sections were stained with Harris' haematoxylin and eosin, dehydrated, cleared in Histo-Clear and mounted in DPX (BDH) resin under coverslips. Slides were viewed and photographed using a compound light microscope equipped with a digital camera.

Acanthamoeba dissemination. In an attempt to determine amoebic dissemination, locusts were injected with $10^{6}$ amoebae in $10 \mu \mathrm{l}$ culture medium as described above. On days 9 and 12 postinjection, locusts were killed and the fat body and flight muscles were dissected out. The isolated tissues were then washed in PBS prior to inoculation onto non-nutrient agar plates seeded with E. coli K-12. To investigate whether amoebae were excreted in the faeces, fresh locust faecal pellets were collected from infected locusts on days 2, 5, 7, 9 and 12 post-injection and inoculated directly onto bacteria-seeded plates.

Feeding activity and weight-change assays. To assess the effects of Acanthamoeba on food consumption, an indirect method was employed where the output of faecal pellets was determined. Locusts were injected intra-abdominally with $10^{6}$ amoebae in $10 \mu \mathrm{l}$ medium or with medium alone, as described above. Subsequently, each locust was kept individually in a cage with a wire-mesh base so that faecal material could be collected from below and did not remain available to the locust (locusts are coprophagic). These cages were kept in a separate holding room at $\sim 30{ }^{\circ} \mathrm{C}$. The weight of the injected locusts was determined at $24 \mathrm{~h}$ intervals, when the faecal material was collected, dried at $37{ }^{\circ} \mathrm{C}$ for $2 \mathrm{~h}$ and weighed.

\section{RESULTS}

\section{Acanthamoeba isolate of the T4 genotype kills locusts}

Locusts in groups of 9 or 10 (total $n=78$ ) were injected with $10^{6}$ amoebae and their mortality was recorded every $24 \mathrm{~h}$ as described above. When kept at room temperature, Acanthamoeba-injected locusts all died within 17 days of infection. Mortality in the infected locusts was significantly greater $(P<0.05$ using a two-sided Fisher's exact test $)$ than the $16 \%$ in locusts receiving sterile culture medium (Fig. 1a). To determine the effects of the inoculum size on mortality, locusts in groups of ten were injected with $10^{3}-10^{6}$ amoebae. Although locust mortality occurred in all groups, it was lowest in the control group (60\% survived to day 29). In the infected locusts, $100 \%$ mortality occurred at all doses, but the speed of kill increased with the size of the inoculum (Fig. 1b): when injected with $10^{3}$ amoebae, it took 29 days for all locusts to die, whereas this was reduced to 17 days with an injection of $10^{6}$ amoebae. All subsequent infection experiments were performed by injecting $10^{6}$ amoebae per locust, and maintaining the infected and control locusts at $\sim 30{ }^{\circ} \mathrm{C}$ in a separate holding room. 
(a)

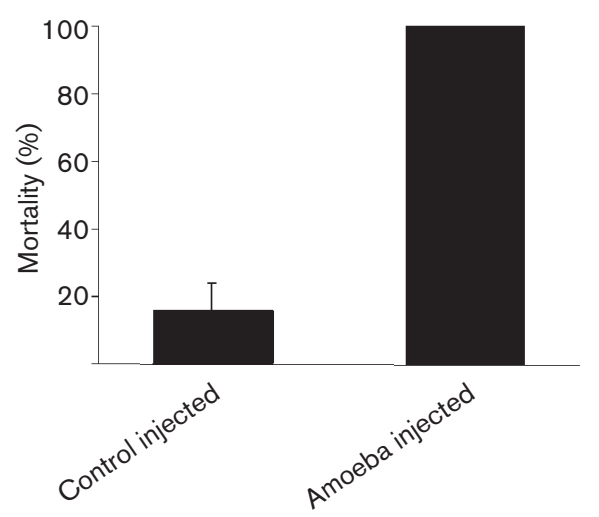

(b)

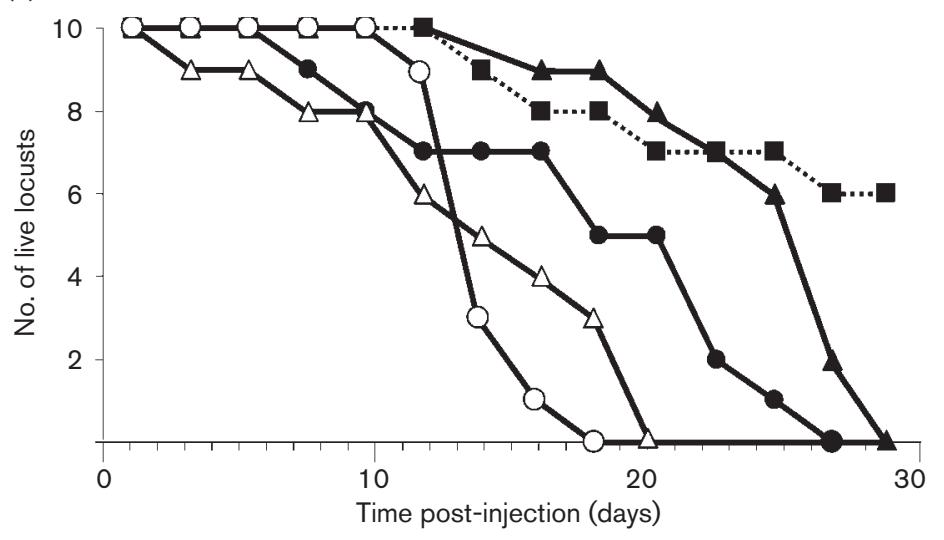

Fig. 1. (a) Locusts in groups of 9 or 10 (total $n=78$ ) injected with $10^{6}$ amoebae all died within 17 days, whereas $84 \%$ of the control locusts $(n=78)$ injected with culture medium alone survived at this time. (b) Representative effects of different numbers of Acanthamoeba injected on locust mortality. Note that Acanthamoeba eventually killed all locusts regardless of the number of amoebae in the inoculum, but the speed of kill depended on the size of the inoculum. $\boldsymbol{\square}$, Control injected; $\boldsymbol{\Delta}, 10^{3}$ amoebae injected; $\bullet, 10^{4}$ amoebae injected; $\triangle, 10^{5}$ amoebae injected; $\bigcirc, 10^{6}$ amoebae injected.

\section{Acanthamoeba invades the locust brain in vivo}

Locusts were injected with $10^{6}$ amoebae and brains were dissected out at $24 \mathrm{~h}$ intervals. Lysates of chlorhexidinetreated brains were positive for viable amoebae in less than $50 \%$ of locusts between days 1 and 4 post-injection. After this time, amoebae were recovered from the brain lysates of all Acanthamoeba-injected locusts (Fig. 2). Of note, brain lysates of amoebae-injected locusts prepared 1-4 days after infection showed the presence of amoebae only after a $48 \mathrm{~h}$ incubation of the plates (Fig. 2b), whereas brain lysates from locusts $>5$ days after infection showed amoebae within $18 \mathrm{~h}$ of incubation (Fig. 2d). By contrast, brain lysates from control locusts did not contain amoebae (Fig. 2a). To demonstrate the potency of chlorhexidine, various concentrations were incubated with $10^{6}$ Acanthamoeba in vitro. The results clearly showed that $20 \mu \mathrm{M}$ chlorhexidine produced $100 \%$ amoebicidal effects (data not shown). To confirm further the presence of amoebae inside the locust brain, histological studies were performed. As shown in Fig. 3, only parasiteinfected locusts showed the presence of amoebae inside the brain. (a)

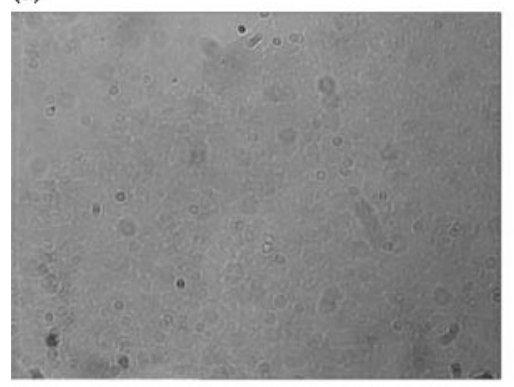

(c)

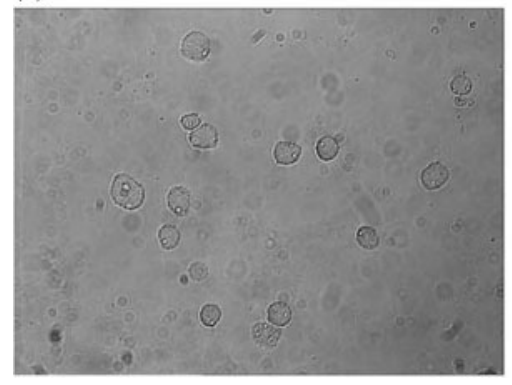

(b)

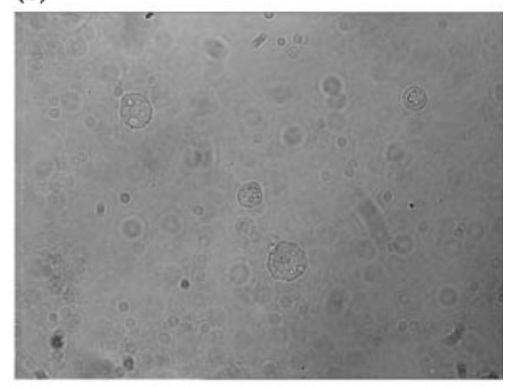

(d)

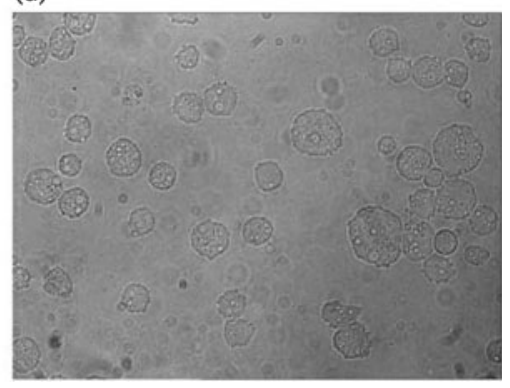

Fig. 2. Acanthamoeba invades the locust brain. Locusts were injected with $10^{6}$ amoebae and their brains were dissected out at $24 \mathrm{~h}$ intervals, which was followed by chlorhexidine treatment to kill extracellular amoebae. On days 1-4 post-injection, less than $50 \%$ of locust brains showed the presence of amoebae. Subsequently, all amoebae-injected locusts showed the presence of viable amoebae $(b-d)$, whilst control locusts were negative (a). Note that brain lysates of locusts on days 1-4 post-injection showed fewer amoebae (b); the number of amoebae increased in number on day 4 (c). From day 5 onwards, there were larger numbers of amoebae in the locust brains (d). The results are representative of three independent experiments. 
(a)

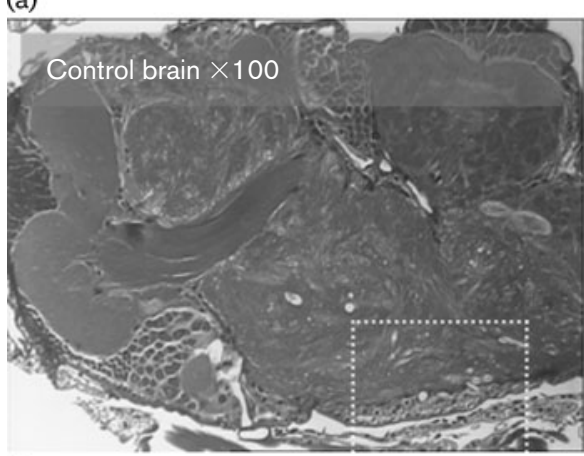

(c)

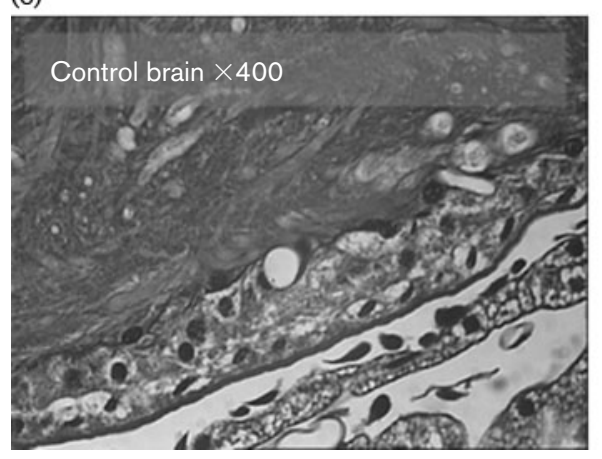

(b)

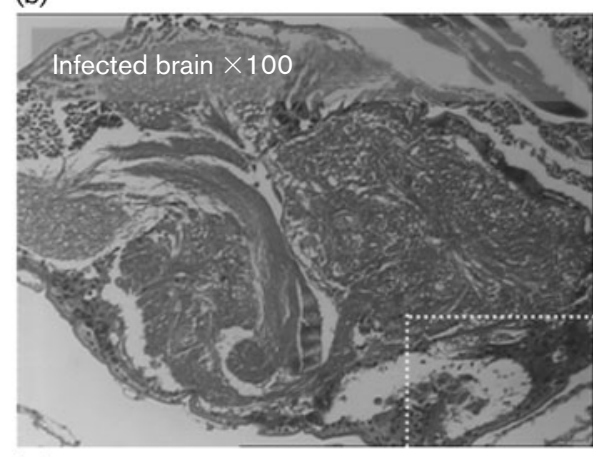

(d)

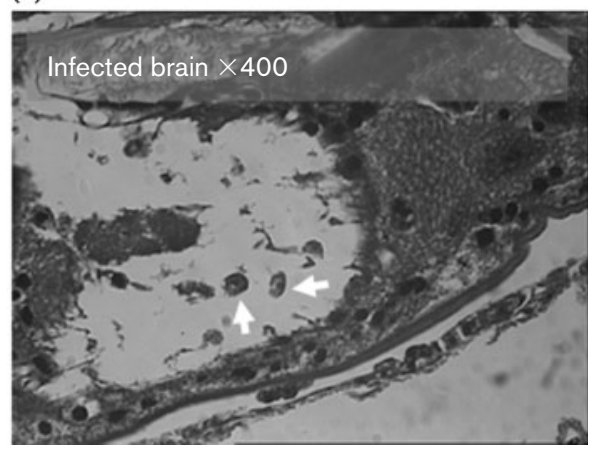

Fig. 3. Representative micrographs of control $(a, c)$ and Acanthamoeba-infected (b, d) locust brains. Locusts were injected with $10^{6}$ amoebae and their brains were dissected out at 7 days post-infection. Subsequently, the brains were sectioned and stained with haematoxylin and eosin. Note that amoebae-injected locusts showed infiltration of the parasites into the brain tissue (d) (indicated by white arrows), whilst control locusts were negative in this respect. The insets in (a) and (b) are shown at higher magnification in (c) and (d), respectively.

\section{Acanthamoeba dissemination within the locust body}

Viable amoebae were isolated from the haemolymph of infected locusts on all days tested (data not shown). Furthermore, viable amoebae were also recovered from the fat body and muscle tissues of injected locusts (data not shown). No amoebae were recovered from the faeces of locusts (data not shown). In samples from control locusts, no amoebae were recovered from haemolymph, the fat body, muscle tissue or faeces.

\section{Acanthamoeba induces sickness behaviour in locusts}

Locusts injected with $10^{6}$ amoebae were monitored for sickness behaviour by monitoring weight change and faeces production. The weight of amoebae-injected locusts appeared to decrease gradually (but this was not statistically significant) with time, until after $\sim 8$ days when there was a significant reduction in weight (Fig. 4). Locust weights were not obtained for later times because $>70 \%$ of the Acanthamoeba-injected locusts died within 9 days at the higher maintenance temperature. These findings were similar to the observations for faeces production. Although there were fluctuations in faecal production in both groups,

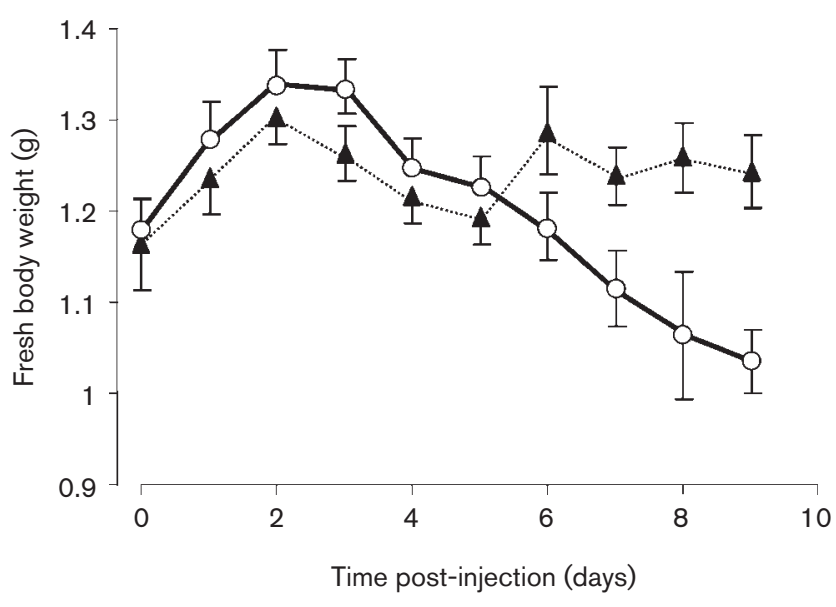

Fig. 4. Acanthamoeba-injected locusts show significant weight reduction after 6-8 days. Day 0 represents the injection day and the values shown are means \pm SEM of the weights of ten locusts. $\bigcirc$, Amoeba injected; $\boldsymbol{\Lambda}$, control injected.

the amoebae-infected locusts showed a gradual decrease with time and a significant reduction from day 4 onwards compared with control medium-injected locusts (Fig. 5). 


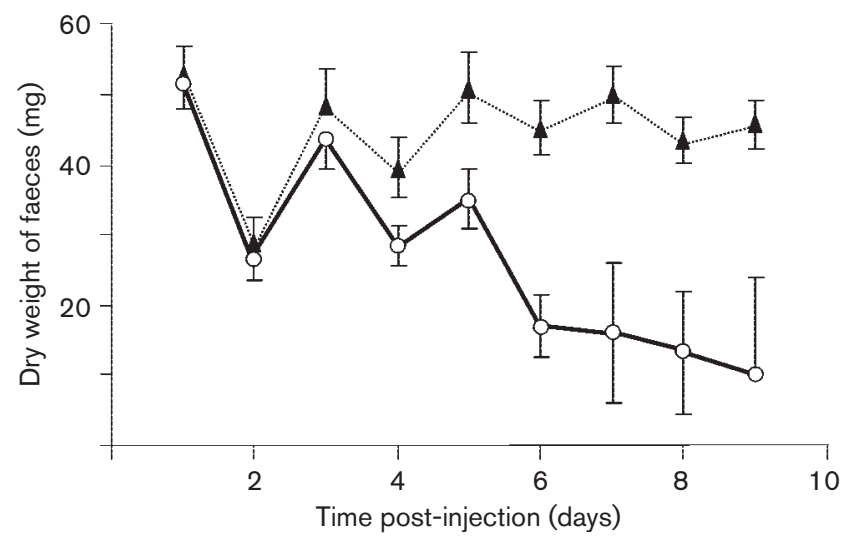

Fig. 5. Acanthamoeba infection decreases faecal production in locusts. The dry weight of faeces of amoebae-injected locusts showed a gradual decrease with time and a significant reduction on day 6 compared with control locusts. Day 0 represents the injection day and the values shown are means \pm SEM of the weights of the dried faeces of live locusts. $\bigcirc$, Amoeba injected; $\boldsymbol{\Delta}$, control injected.

\section{DISCUSSION}

An incomplete understanding of the pathogenesis and pathophysiology of Acanthamoeba encephalitis has resulted in significant morbidity and mortality. In part, this is due to the lack of availability of a rapid, reproducible and relatively easy-to-perform in vivo model for the study of the process of amoebae invasion of the CNS. Here, for what is believed to be the first time, it was shown that locusts can be used as a novel model for the in vivo study of CNS infection by Acanthamoeba spp. The remarkable parallels between the innate immune responses of mammals and insects (Scully \& Bidochka, 2006), especially as insects possess a highly selective blood-brain barrier exhibiting functional properties comparable to those of vertebrates (Carlson et al., 2000), and the fact that Acanthamoeba encephalitis is limited mostly to immunocompromised populations, suggest that locusts could make a useful model in which to study the pathogenesis of Acanthamoeba.

It is widely accepted that haematogenous spread is a prerequisite for invasion of the CNS by Acanthamoeba and that amoebae most likely enter at the sites of the bloodbrain barrier (Martinez et al., 1975; Martinez, 1985, 1991). In support of this, haematoxylin/eosin-stained sections of the brain tissue of Acanthamoeba encephalitis patients exhibit large numbers of amoebae in the perivascular space. By contrast, pathogens that enter via the choroid plexus route will end up in the cerebrospinal fluid. Thus, the pathology in such cases will appear first in the ventricles, and only at later stages with extensive inflammation will it involve the brain parenchyma, a finding that is normally inconsistent with Acanthamoeba encephalitis pathological features and further suggests that traversal of the bloodbrain barrier by amoebae most likely occurs at the cerebral capillary endothelium. Affected tissues other than the CNS may include subcutaneous tissue, skin, liver, lungs, kidneys, adrenal glands, pancreas, prostate, lymph nodes and bone marrow, which further suggest haematogenous spread. Similarly, in the locust, amoebae were recovered from locust haemolymph, as well as other tissues such as the fat body and muscles (but not faeces) on all days tested during the course of infection, suggesting that amoebae produce parasitaemia and survive the onslaught of the locust's innate immune defences. Here, the term 'parasitaemia' is used to describe simply the presence of amoebae in the locust haemolymph. Several approaches have been tested in an attempt to determine the presence of amoebae in haemolymph quantitatively, but so far these have proved unsuccessful and future studies will address this problem. In addition, histological studies are in progress to determine the precise localization of amoebae within the brain tissues. Of note, although our findings show the presence of amoebae in the brain, the mechanisms of amoeba crossing the locust blood-brain barrier remain incompletely understood. Our findings that amoebae are not recovered from locusts' faeces are in contrast to the findings of Zaman et al. (1999) who discovered Acanthamoeba cysts in human faeces. The fact that both humans and locusts encounter amoebae in their natural environment but only humans show the presence of amoebae in faeces is interesting and requires further investigation. Overall, these findings suggest that Acanthamoeba infection of the various tissues and the presence of amoebae in faeces are independent events, as observed by Zaman et al. (1999). However, it should be noted that there has been a single report involving Acanthamoeba in a gastrointestinal tract infection in a patient suffering from pyloric perforation, which was biopsied, and demonstrated the presence of Acanthamoeba trophozoites and cysts (Thamprasert et al., 1993).

It is noteworthy that injection of amoebae caused total mortality with as few as $10^{3}$ amoebae, but the speed of kill was increased significantly with higher inoculum sizes and an increased maintenance temperature. Regardless of the various inocula, amoebae were present in the brain lysates of Acanthamoeba-injected locusts, and it is tempting to speculate that Acanthamoeba mediate locust death in part by invading the locust CNS. Given that the molecular components of the blood-brain barrier in insects such as Drosophila are homologous to those of vertebrates (Willott et al., 1993), it is possible that there will be similarities between the mechanisms by which Acanthamoeba invades the CNS of mammals and that of locusts, and studies are in progress to test this.

Another interesting finding was that Acanthamoeba-injected locusts displayed pronounced weight loss compared with the control group. The significant weight loss slightly preceded by reduced faeces production seen in locusts injected with amoebae suggests that this aspect of sickness behaviour may result from reduced sensory input.

Previous studies have identified several factors associated with the virulence of Acanthamoeba, including mannose- 
binding protein expressed on the plasma membrane surface of amoebae, serine proteases, phospholipases and ectoATPases (Khan, 2006). By studying the role of these factors in vivo, the validity of the locust model will be tested further. Nevertheless, the results of the experiments described here support strongly the proposal that an insect model can mimic human Acanthamoeba encephalitis and can elicit a full spectrum of host-pathogen interactions. It is anticipated that the probable existence of additional virulence determinants of the amoeba can be investigated in vivo using this insect system.

\section{ACKNOWLEDGEMENTS}

We gratefully acknowledge Mary Lightfoot for her experienced, skilful technical assistance and for providing large numbers of healthy locusts, and Mohamed Mohamud who helped with some of the experiments in this study.

\section{REFERENCES}

Carlson, S. D., Juang, J. L., Hilgers, S. L. \& Garment, M. B. (2000). Blood barriers of the insect. Annu Rev Entomol 45, 151-174.

Goldsworthy, G., Mullen, L., Opoku-Ware, K. \& Chandrakant, S. (2003a). Interactions between the endocrine and immune system in locusts. Physiol Entomol 28, 54-61.

Goldsworthy, G., Chandrakant, S. \& Opoku-Ware, K. (2003b). Adipokinetic hormone enhances nodule formation and phenoloxidase activation in adult locusts injected with bacterial lipopolysaccharide. J Insect Physiol 49, 795-803.

Khan, N. A. (2006). Acanthamoeba: biology and increasing importance in human health. FEMS Microbiol Rev 30, 564-595.

Khan, N. A. (2007). Acanthamoeba invasion of the central nervous system. Int J Parasitol 37, 131-138.
Khan, N. A. \& Goldsworthy, G. (2007). Novel model to study virulence determinants of Escherichia coli K1. Infect Immun 75, 5735-5739.

Khan, N. A., Jarroll, E. L., Panjwani, N., Cao, Z. \& Paget, T. A. (2000). Proteases as markers of differentiation of pathogenic and nonpathogenic Acanthamoeba. J Clin Microbiol 38, 2858-2861.

Marciano-Cabral, F. \& Cabral, G. (2003). Acanthamoeba spp. as agents of disease in humans. Clin Microbiol Rev 16, 273-307.

Martinez, A. J. (1985). Free-Living Amoebas: Natural History, Prevention, Diagnosis, Pathology and Treatment of Disease. Boca Raton, FL: CRC Press.

Martinez, A. J. (1991). Infections of the central nervous system due to Acanthamoeba. Rev Infect Dis 13, S399-S402.

Martinez, A. J., Markowitz, S. M. \& Duma, R. J. (1975). Experimental pneumonitis and encephalitis caused by Acanthamoeba in mice: pathogenesis and ultrastructural features. J Infect Dis 131, 692-699.

Mullen, L. \& Goldsworthy, G. (2003). Changes in lipophorins are related to the activation of phenoloxidase in the haemolymph of Locusta migratoria in response to injection of immunogens. Insect Biochem Mol Biol 33, 661-670.

Schuster, F. L. (2002). Cultivation of pathogenic and opportunistic free-living amoebas. Clin Microbiol Rev 15, 342-354.

Schuster, F. L. \& Visvesvara, G. S. (2004). Free-living amoebae as opportunistic and non- opportunistic pathogens of humans and animals. Int J Parasitol 34, 1001-1027.

Scully, L. R. \& Bidochka, M. J. (2006). Developing insect models for the study of current and emerging human pathogens. FEMS Microbiol Lett 263, 1-9.

Thamprasert, K., Khunamornpong, S. \& Morakote, N. (1993). Acanthamoeba infection of peptic ulcer. Ann Trop Med Parasitol 87, 403-405.

Willott, E., Balda, M. S., Fanning, A. S., Jameson, B., Van Itallie, C. \& Anderson, J. M. (1993). The tight junction protein $\mathrm{ZO}-1$ is homologous to the Drosophila discs-large tumor suppressor protein of septate junctions. Proc Natl Acad Sci U S A 90, 7834-7838.

Zaman, V., Zaki, M. \& Manzoor, M. (1999). Acanthamoeba in human faeces from Karachi. Ann Trop Med Parasitol 93, 189-191. 